Stefanie Schwerdtfeger, Mathias Wilde, Martin Lanzendorf

\title{
Motive des Fahrens ohne (gültigen) Fahrschein
}


In den Arbeitspapieren zur Mobilitätsforschung veröffentlichen wir Ergebnisse aus Forschung und Lehre der Goethe-Universität.

Der Bericht wurde innerhalb des Projektes „Fahren ohne (gültigen) Fahrschein - Motive, Akzeptanz und Handlungsoptionen im Gebiet des Rhein-Main-Verkehrsverbundes" erarbeitet und repräsentiert das zweite Arbeitspaket. Das Forschungsprojekt wurde mit großzügiger Unterstützung des Rhein-Main-Verkehrsverbundes (RMV) finanziert. Die Bearbeitung erfolgte in enger Kooperation mit dem RMV und der Rhein-Main-Verkehrsverbund Servicegesellschaft $\mathrm{mbH}$ (rms). Die Verantwortung für den Inhalt dieser Veröffentlichung liegt ausschließlich bei den Autor/innen.

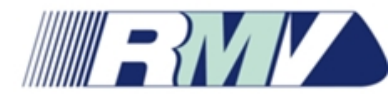

RHEIN-MAIN-VERKEHRSVERBUND

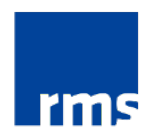

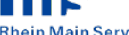

Impressum

Arbeitsgruppe Mobilitätsforschung

Prof. Dr. Martin Lanzendorf

Institut für Humangeographie

Goethe-Universität Frankfurt am Main

Campus Westend

Theodor-W.-Adorno-Platz 6, PEG

D-60629 Frankfurt am Main

Email: mobilitaetsforschung@uni-frankfurt.de

Tel.: $\quad$ +49 (0)69-798-35179

ISSN: 2363-8133

urn:nbn:de:hebis:30:3-403157

Arbeitspapiere zur Mobilitätsforschung Nr. 16 (2018)

Redaktion: Dr. Hannah Müggenburg

Zitierweise: Schwerdtfeger, S; Wilde, Mathias; Lanzendorf, Martin (2018): Motive des Fahrens ohne (gültigen) Fahrschein. Arbeitspapiere zur Mobilitätsforschung Nr. 16. Frankfurt a.M. 
Stefanie Schwerdtfeger, Mathias Wilde, Martin Lanzendorf

\section{Motive des Fahrens ohne (gültigen) Fahrschein}





\section{Inhaltsverzeichnis}

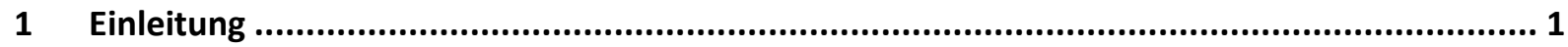

2 Die Untersuchung des Fahrens ohne (gültigen) Fahrschein mit Methoden der qualitativen

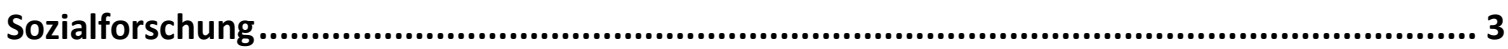

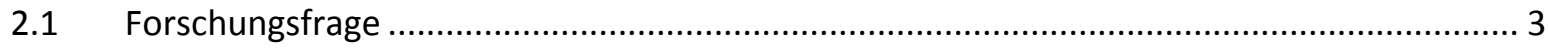

2.2 Qualitative Interviews zur Untersuchung des Fahrens ohne (gültigen) Fahrschein .............. 3

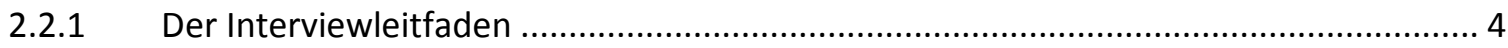

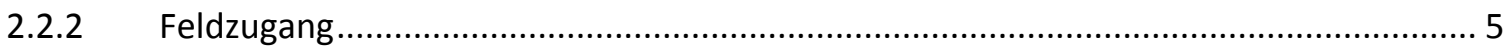

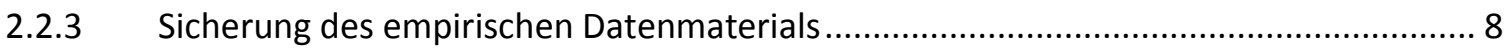

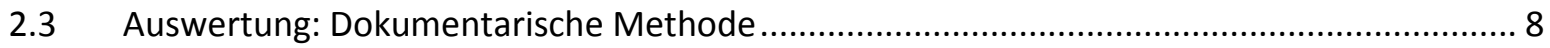

3 Motive des Fahrens ohne (gültigen) Fahrschein .................................................................. 9

3.1 Nicht-intentionales Fahren ohne (gültigen) Fahrschein..................................................... 10

3.1.1 Motivtyp: Personen ohne Tickets.............................................................................. 10

3.1.2 Motivtyp: Personen, die ihre Zeitkarten vergessen haben ........................................... 11

3.1.3 Motivtyp: Personen mit falschen Tickets .................................................................... 12

3.2 Intentionales Fahren ohne (gültigen) Fahrschein .......................................................... 14

3.2.1 Motivtyp: Rational und opportunistisch Handelnde ................................................... 14

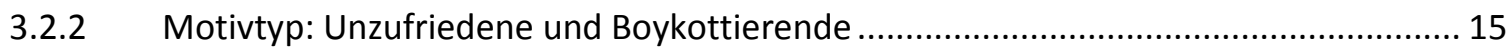

3.2.3 Motivtyp: Mobilitätsarme und sozial Exkludierte ........................................................ 16

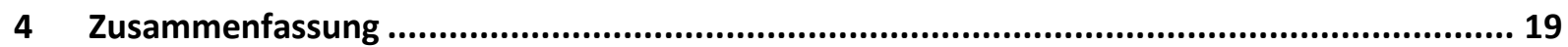

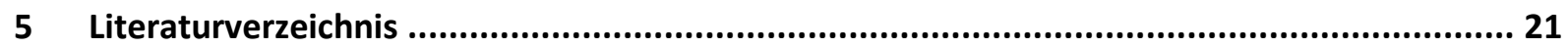

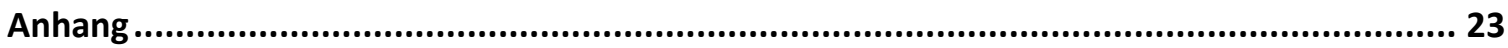

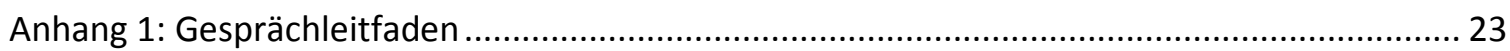

Anhang 2: Fragebogen zur Erhebung soziodemographischer Charakteristika ............................. 27 



\section{Abbildungsverzeichnis}

Abbildung 1: Fragekategorien des Interviewleitfadens .

4

Abbildung 2: Übersicht der Befragungsorte im RMV-Verbundgebiet

Abbildung 3: Typologie des Fahrens ohne (gültigen) Fahrschein

\section{Tabellenverzeichnis}

Tabelle 1: Übersicht der Interviewteilnehmenden 6

Tabelle 2: Zusammensetzung der Typologie des Fahrens ohne (gültigen) Fahrschein. 9

Tabelle 3: Typologie des Fahrens ohne (gültigen) Fahrschein 20

\section{Abkürzungsverzeichnis}

DB

Deutsche Bahn

EBE

erhöhtes Beförderungsentgelt

ÖPNV

Öffentlicher Personennahverkehr

RMV

Rhein-Main-Verkehrsverbund

VGF

Stadtwerke Verkehrsgesellschaft Frankfurt am Main 



\section{Einleitung}

Das Fahren ohne (gültigen) Fahrschein in den Verkehrsmitteln des Öffentlichen Personennahverkehrs (ÖPNV) stellt bereits seit den 1960er Jahren ein Problem für Verkehrsunternehmen dar. Aufgrund von Sparmaßnahmen wurde das damals beschäftigte Fahrkartenpersonal - zunächst in Europa und kurze Zeit später in den USA - durch Fahrkartenautomaten ersetzt (Deibel 1981; van Andel 1989; Barabino et al. 2013, 2014). Die Verantwortlichkeit für den Besitz einer gültigen Fahrkarte verlagerte sich somit vom Personal auf die Fahrgäste, die seither die Möglichkeit haben, den ÖPNV auch ohne (gültiges) Ticket nutzen zu können (van Andel 1989; Yin et al. 2012). Während also die Personalkosten gesunken sind, ist der Anteil der Personen, die den ÖPNV ohne (gültigen) Fahrschein benutzen, gestiegen (z.B. Boyd et al. 1989; Smith \& Clarke 2000; Killias et al. 2009). In anderen Worten: „As machines replaced staff, however, fare evasion increased, in buses, trams and trains" (Bijleveld 2007: 185). Durch die Benutzung des ÖPNV ohne (gültige) Fahrkarte gehen den Verkehrsunternehmen Einnahmen verloren (Bonfanti \& Wagenknecht 2010; Clarke et al. 2009). Auch wird auf den Schaden für das Image des ÖPNV verwiesen und es wird befürchtet, dass zahlende Fahrgäste - z.B. im Fall von Aggressivität, die in Verbindung mit dem Fahren ohne (gültigen) Fahrschein auftreten kann - auf andere Verkehrsmittel umsteigen (Bijleveld 2007; Barabino et al. 2013, 2014).

Aber auch für die ÖPNV-Nutzenden stellt das Fahren ohne (gültigen) Fahrschein ein Problem dar, da Personen, die den ÖPNV ohne (gültiges) Ticket benutzen, in Deutschland eine Straftat begehen. Laut §265a StGB gilt u.a. das Fahren ohne (gültigen) Fahrschein als Erschleichung von Leistungen, was unter Betrugs- und Untreuedelikten subsummiert wird und zu den Vermögensdelikten zählt. Verkehrsunternehmen sind dazu berechtigt, das Fahren ohne (gültigen) Fahrschein zur Anzeige zu bringen. Vielerorts finden Gerichtsverhandlungen mit Personen statt, die den ÖPNV mehrmals ohne (gültiges) Ticket benutzt haben. In den Urteilen werden zumeist Geldstrafen, aber auch Sozialstunden und Haftstrafen verhängt.

Obwohl das Fahren ohne (gültigen) Fahrschein bereits seit Ende der 1980er Jahre ein Thema in wissenschaftlichen Studien ist, wurde die Frage nach den Motiven und Beweggründen, die dem Fahren ohne (gültigen) Fahrschein zugrunde liegen, bislang nur in Ansätzen thematisiert. Der Stand der Forschung zeigt, dass der Fokus bislang hauptsächlich auf betriebswirtschaftlichen Aspekten lag. Daneben setzen sich auch die Rechtswissenschaften und die Kriminologie mit dem Thema auseinander. Einige wenige Studien verfolgen darüber hinaus einen sozialwissenschaftlichen Ansatz. Die Beweggründe des Fahrens ohne (gültigen) Fahrschein werden in den vorhandenen Studien lediglich objektiv betrachtet (Schwerdtfeger et al. 2016). Insgesamt zeigt der Stand der Forschung, dass eine tiefgehende sozialwissenschaftliche Untersuchung der Motive und Beweggründe des Fahrens ohne (gültigen) Fahrschein bislang nicht vorliegt. Die Ergebnisse aus den bereits publizierten Studien zeigen, dass weiterer Forschungsbedarf hinsichtlich der Motive und Beweggründe notwendig ist (z.B. ebd.; Guarda et al. 2016).

Dies spiegelt sich auch in den Maßnahmen wider, die von Verkehrsunternehmen und -verbünden umgesetzt wurden. Eine Analyse der Best-Practice-Maßnahmen hat ergeben, dass das Potenzial der realisierten Maßnahmen oder Praktiken in Bezug auf eine nachhaltige Senkung der Quote an Personen ohne (gültiges) Ticket gering ist. Temporär begrenzte Einzelmaßnahmen führen bei der Reduzierung des Fahrens ohne (gültigen) Fahrschein zwar zu einem kurzfristigen Effekt, allerdings können keine Maßnahmen mit einem dauerhaften Erfolg verzeichnet werden (Schwerdtfeger et al. 2017). Aus diesen Gründen ist es aus Sicht der Verkehrsunternehmen und -verbünde wichtig, 
die Motive des Fahrens ohne (gültigen) Fahrschein zu verstehen. Eine solche Perspektive erweitert das Handlungsspektrum und lädt dazu ein, neue Maßnahmen und Praktiken zur Reduzierung des Fahrens ohne (gültigen) Fahrschein zu generieren sowie zu testen, was letztendlich zum Ziel hat, den ÖPNV dauerhaft und sozial gerecht finanzieren zu können.

Das vorliegende Arbeitspapier zeigt die Ergebnisse der qualitativen Untersuchung der Motive des Fahrens ohne (gültigen) Fahrschein im Bediengebiet des Rhein-Main-Verkehrsverbundes (RMV). Das Ziel der Arbeit ist ein tiefgehendes und umfangreiches Verständnis der Motive des Fahrens ohne (gültigen) Fahrschein. Zu diesem Zweck wurden qualitative Interviews mit Personen durchgeführt, die ohne (gültigen) Fahrschein in den Fahrzeugen des ÖPNV angetroffen wurden. Als Ergebnis wurden Typen gebildet, die das Feld repräsentieren.

Nachdem der Untersuchungsgegenstand und das Ziel des Arbeitspapieres in diesem Kapitel beschrieben wurden, folgt in Kapitel 2 eine Erläuterung des Forschungsdesigns. Es wird erklärt, welche Forschungsfrage der Untersuchung zugrunde liegt (Kapitel 2.1), wie die qualitativen Interviews konzipiert wurden (Kapitel 2.2) und schließlich, wie die Interviews ausgewertet wurden (Kapitel 2.3). Daran anschließend erfolgt in Kapitel 3 eine Erläuterung der empirischen Ergebnisse, indem sechs Typen des Fahrens ohne (gültigen) Fahrschein vorgestellt werden. Eine Zusammenfassung des Arbeitspapieres ist in Kapitel 4 zu finden. 


\section{Die Untersuchung des Fahrens ohne (gültigen) Fahrschein mit Methoden der qualitativen Sozialforschung}

Über die Motive des Fahrens ohne (gültigen) Fahrschein ist bislang wenig bekannt. In einem klassischen Forschungsdesign über lebensweltliche Phänomene bietet sich in solchen Fällen an, ein Verständnis über ein Phänomen - wie hier die Motivlage - über ein qualitatives Verfahren zu erlangen (Guarda et al. 2016). Im Mittelpunkt der Untersuchung stehen Personen, die den ÖPNV ohne (gültigen) Fahrschein benutzen: Sie können als AlltagsexpertInnen verstanden werden und Auskunft über ein noch weitgehend unerforschtes Feld geben.

Zur Exploration der Motive des Fahrens ohne (gültigen) Fahrschein wurden qualitative Interviews mit Personen durchgeführt, die bei einer Fahrkartenkontrolle keine (gültige) Fahrkarte vorweisen konnten. Das Ziel war die Analyse der Motive des Fahrens ohne (gültigen) Fahrschein und eine Typenbildung, welche durch die Auswertung des Interviewmaterials mithilfe der dokumentarischen Methode (Kapitel 2.3) gebildet wurde.

\subsection{Forschungsfrage}

Das Fahren ohne (gültigen) Fahrschein wurde bereits aus unterschiedlichen wissenschaftlichen Disziplinen und Perspektiven heraus untersucht. Die Studien befassen sich hauptsächlich mit objektiven Determinanten, vor allem aus der Perspektive der Rechts- und Betriebswissenschaften sowie aus einer kriminologischen Perspektive. Einige wenige Studien, die allerdings kein tiefgehendes Verständnis des Fahrens ohne (gültigen) Fahrschein vermitteln, verfolgen zudem einen sozialwissenschaftlichen Ansatz (Schwerdtfeger et al. 2016). Die vorliegende Untersuchung geht darüber hinaus und versucht mit der Beantwortung folgender Fragestellung ein tieferes Verständnis über die Motive des Fahrens ohne (gültigen) Fahrschein zu gewinnen: Welche Motive liegen der Benutzung des ÖPNV ohne (gültigen) Fahrschein zugrunde?

Motive wirken handlungsbeeinflussend, weswegen das Fahren ohne (gültigen) Fahrschein als bewusste Handlung verstanden werden kann (vgl. Kapitel 3). Die Fragestellung zielt also auf Handlungshintergründe und soll Antworten dazu liefern, inwiefern das Fahren ohne (gültigen) Fahrschein beispielsweise politisch oder finanziell bedingt ist oder ob es zweckrationalen Handlungslogiken entspringt. Schließlich sollen die unterschiedlichen Motive in einer Typologie zusammengefasst werden, die als Ausgangspunkt für die Formulierung von Handlungsfeldern in Bezug auf das Fahren ohne (gültigen) Fahrschein dient.

\subsection{Qualitative Interviews zur Untersuchung des Fahrens ohne (gültigen) Fahrschein}

Die Interviews im Forschungsprojekt wurden als problemzentrierte Interviews nach Witzel (1982) durchgeführt. Im Gegensatz zum rein induktiven narrativen Interview, ist das problemzentrierte Interview durch eine Wechselwirkung von induktiven und deduktiven Schritten gekennzeichnet (Lamnek 2010: 332 f.; Misoch 2015: 71 ff.). Für das Fahren ohne (gültigen) Fahrschein bedeutet dies, dass die vorab gewonnenen theoretischen Erkenntnisse ebenso in die empirische Untersuchung eingehen wie neu gewonnene Erkenntnisse, die aus den Interviews selbst 
hervorgegangen sind. Der Forschungsprozess ist folglich nicht starr und linear. Vielmehr kann der Interviewleitfaden angepasst und verändert und somit der Forschungsprozess kontinuierlich auf den Untersuchungsgegenstand gelenkt werden (Misoch 2015: 71).

\subsubsection{Der Interviewleitfaden}

Der Gesprächsleitfaden (Anhang 1) wurde basierend auf den Forschungslücken aus dem Stand der Forschung zum Fahren ohne (gültigen) Fahrschein (vgl. Schwerdtfeger et al. 2016) entwickelt und besteht aus insgesamt fünf Fragekategorien (Abbildung 1): (1) Gründe des Fahrens ohne (gültigen) Fahrschein, (2) individuelle Merkmale der Lebenswirklichkeit, (3) Aspekte individueller Mobilität, (4) Einstellungen gegenüber dem ÖPNV sowie (5) individuelle Meinungen und Wertungen.

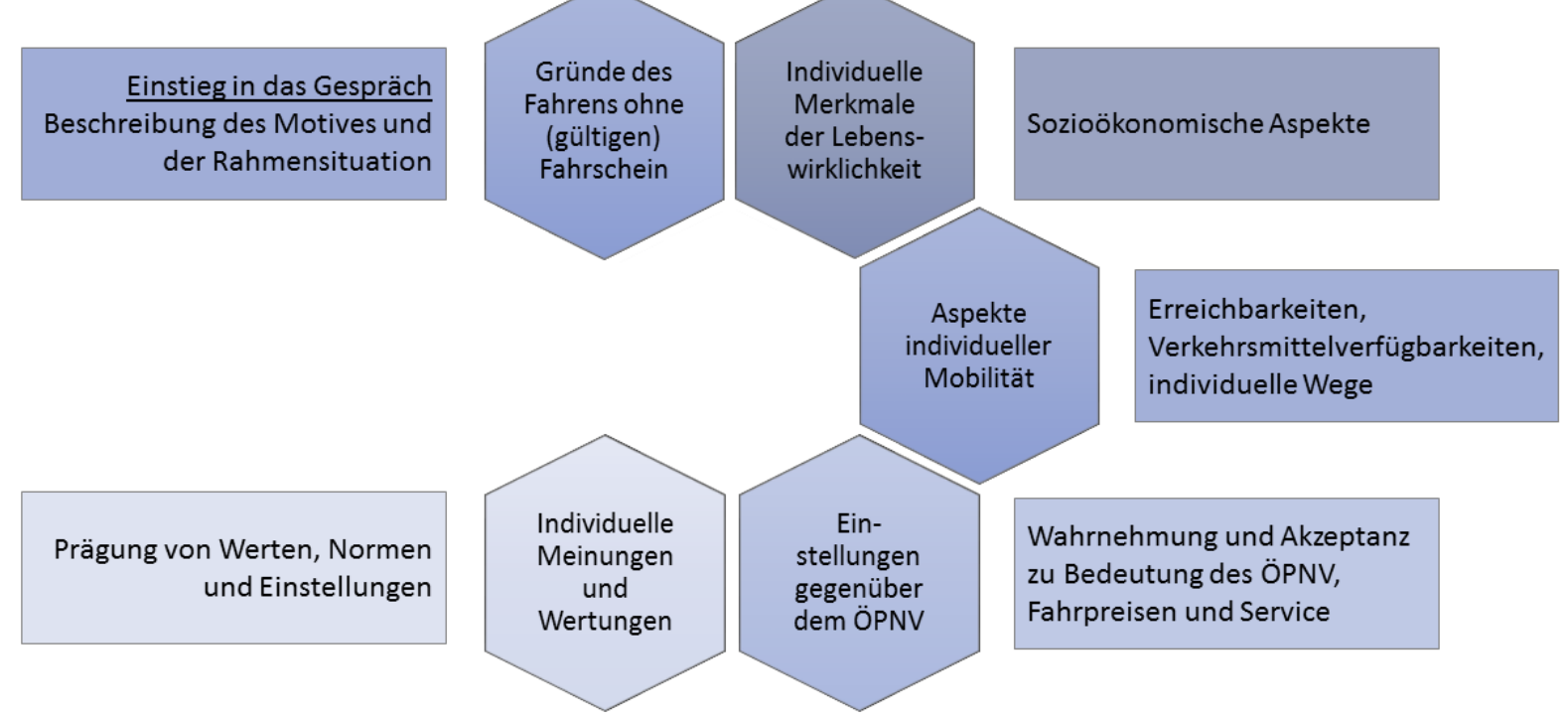

Abbildung 1: Fragekategorien des Interviewleitfadens Quelle: Eigene Darstellung

Die Fragekategorie Gründe des Fahrens ohne (gültigen) Fahrschein beinhaltet das zentrale Thema der Befragung. Diese Fragekategorie diente insbesondere dem Gesprächseinstieg, da sie bereits über die erste Frage eine „narrative Gesprächsstruktur“ (Witzel 1982: 96) vermittelt. So wurden die Interviewteilnehmenden zunächst gebeten, die Situation und die Gründe sowie Regelmäßigkeiten bezüglich des Fahrens ohne (gültigen) Fahrschein zu schildern.

Die Fragekategorie Individuelle Merkmale der Lebenswirklichkeit bezieht sich auf sozioökonomische Aspekte, wie etwa Einkommen und Haushaltsgröße oder den beruflichen Status. Somit lassen sich erste Rückschlüsse auf die Motive des Fahrens ohne (gültigen) Fahrschein ziehen. Wurde beispielsweise vorab argumentiert, dass zu hohe Preise für das Fahren ohne (gültigen) Fahrschein verantwortlich seien, so kann unter Heranziehung sozioökonomischer Aspekte eine Einschätzung diesbezüglich erfolgen.

Die Fragekategorie Aspekte individueller Mobilität soll Rückschlüsse auf die Bedeutung der ÖPNVNutzung liefern. So wurden hier Fragen gestellt, die sich zum Beispiel auf die Häufigkeit der ÖPNV- 
Nutzung oder die Verkehrsmittelverfügbarkeit und -nutzung beziehen. Mit dieser Kategorie soll eingeschätzt werden, wie stark oder schwach die Erläuterung eines Motivs in Bezug auf das individuelle Mobilsein der befragten Person erscheint.

Dieselbe Funktion hat die Fragekategorie Einstellungen gegenüber dem ÖPNV. Hier sollen Symboliken und Bedeutungen erfahren werden, die Motive verstärken oder abmildern können. Ein Beispiel dafür ist, inwiefern der ÖPNV als bedeutsam für Mensch und Umwelt bewertet wird.

Mit der Fragekategorie individuelle Meinungen und Wertungen wurde das Bild abgefragt, welches die befragten Personen zum Fahren ohne (gültigen) Fahrschein entwerfen: Wird es beispielsweise als Straftat oder eher als Bagatelle eingestuft? In dieser Fragekategorie liegt der Fokus ebenso auf Werten und Normen, die durch die befragten Personen vertreten oder missachtet werden.

Jeweils im Anschluss an das Interview wurde den Personen ein Kurzfragebogen zur Erhebung sozio-demographischer Merkmale ausgehändigt (Anhang 2). Neben Geschlecht und Alter wurden haushaltsbezogene Informationen wie das monatliche Nettoeinkommen und die Haushaltsgröße abgefragt.

\subsubsection{Feldzugang}

Zur Befragung von Personen, die den ÖPNV ohne (gültiges) Ticket benutzen, wurden FahrkartenkontrolleurInnen im RMV-Gebiet mehrmals während ihrer Arbeitszeiten begleitet. Von September bis Mitte Dezember 2015 wurde das Fahrkartenprüfpersonal der Deutschen Bahn (DB) auf den S-Bahnen, der Stadtwerke Verkehrsgesellschaft Frankfurt am Main (VGF) bei Stationskontrollen und das RMV-Service-Team, das auf unterschiedlichen Verkehrsmitteln im gesamten RMV-Gebiet eingesetzt wird, begleitet. So konnten unterschiedliche öffentliche Verkehrsmittel - von der Großstadt Frankfurt bis hin zum ländlichen Raum - innerhalb des RMVGebietes begleitet werden (Abbildung 2).

Damit die Situation der Fahrkartenkontrolle so natürlich wie möglich verlaufen konnte, hat sich die Forscherin zunächst im Hintergrund gehalten. Während der Ausstellung des erhöhten Beförderungsentgeltes durch die Fahrausweisprüfenden wurden die Personen, die ohne (gültiges) Ticket angetroffen wurden, über die Möglichkeit zur Teilnahme an der Befragung informiert. In diesem Zuge wurde erwähnt, dass Teilnehmende nach Abschluss des Gespräches ein Incentive in Höhe von 60 Euro erhalten. Erst nach der Interessensbekundung durch die Personen, denen ein erhöhtes Beförderungsentgelt (EBE) ausgestellt wurde, ist die Forscherin in den Vordergrund getreten und hat mit der Kontaktaufnahme und dem Vorgespräch zum Interview begonnen. Dabei wurde auf eine klare Abgrenzung der Forscherin zu den Kontrolleurlnnen geachtet, sodass die potenziellen InterviewpartnerInnen nicht das Gefühl bekommen, vom Verkehrsunternehmen beobachtet zu werden. Außerdem verhalf diese Abgrenzung zu der Schaffung einer Vertrauensdimension. Darüber hinaus wurde in dieser ersten Kontaktphase auf die Einhaltung des Datenschutzes hingewiesen und das weitere Vorgehen erläutert. Nach erneuter Zustimmung wurde das Verkehrsmittel bei den Kontrollen im Verkehrsmittel zusammen mit dem Fahrkartenprüfpersonal verlassen und ein geeigneter Ort zur Durchführung des Gespräches gesucht. Lediglich in zwei Fällen konnte das Interview direkt im Verkehrsmittel stattfinden, da sich keine weiteren Fahrgäste darin befanden. Bei den Stationskontrollen wurde ein Ort in der Nähe der kontrollierten Station gesucht. Die Interviews fanden ausschließlich zwischen der Forscherin und der jeweiligen Gesprächsperson statt - das Fahrkartenprüfpersonal hat nicht am Interview teilgenommen (vgl. die Übersicht der TeilnehmerInnen an der Befragung in Tabelle 1). 


\begin{tabular}{|c|c|c|c|c|c|c|c|}
\hline Interview \# & Geschlecht & Alter [Jahre] & Staatsangehörigkeit & Berufsbezeichnung & monatliches Nettoeinkommen & Interview durchgeführt & Ort \\
\hline 1 & $\mathrm{~m}$ & 28 & französisch & Bankangestellter & 3.000 bis unter $4.000 €$ & U-Bahn, Stationskontrolle & FFM, Kirchplatz \\
\hline 2 & $\mathrm{~m}$ & 32 & deutsch & Industrie-Angestellter & 1.000 bis unter $2.000 €$ & Bus & Hain-Gründau \\
\hline 3 & $\mathrm{~m}$ & 26 & türkisch & Elektromonteur & 1.000 bis unter $2.000 €$ & HLB & Grävenwiesbach \\
\hline 4 & $\mathrm{~m}$ & 58 & deutsch & Landwirtschaftshelfer & 1.000 bis unter $2.000 €$ & HLB & Usingen \\
\hline 5 & $\mathrm{~m}$ & 59 & italienisch & --- & unter $500 €$ & HLB & Bad Homburg \\
\hline 6 & $\mathrm{~m}$ & 22 & deutsch & Student & 500 bis unter $1.000 €$ & HLB & Usingen \\
\hline 7 & $\mathrm{~m}$ & 21 & deutsch & Gastronomie-Mitarbeiter & 500 bis unter $1.000 €$ & U-Bahn, Stationskontrolle & FFM, BornheimMitte \\
\hline 8 & w & 27 & deutsch & Rechtsreferendarin & 2.000 bis unter $3.000 €$ & U-Bahn, Stationskontrolle & FFM, BornheimMitte \\
\hline 9 & $w$ & 22 & deutsch & Schülerin & unter $500 €$ & U-Bahn, Stationskontrolle & FFM, BornheimMitte \\
\hline 10 & $\mathrm{~m}$ & 28 & deutsch & Informationselektroniker & 500 bis unter $1.000 €$ & S-Bahn & FFM, Griesheim \\
\hline 11 & $w$ & 18 & deutsch & Schülerin & unter $500 €$ & S-Bahn & FFM, Höchst \\
\hline 12 & w & 32 & deutsch & Buchhalterin & 1.000 bis unter $2.000 €$ & U-Bahn, Stationskontrolle & FFM, Höhenstraße \\
\hline 13 & $\mathrm{~m}$ & 48 & spanisch & Weiterbildungsberater & 1.000 bis unter $2.000 €$ & U-Bahn, Stationskontrolle & FFM, Höhenstraße \\
\hline 14 & $\mathrm{~m}$ & 25 & deutsch & Fachkraft Lagerlogistik & 2.000 bis unter $3.000 €$ & Bus & Offenbach, Sprendlinger Landstraße \\
\hline 15 & $\mathrm{~m}$ & 57 & niederländisch & IT-Consultant & $4.000 €$ und mehr & U-Bahn, Stationskontrolle & FFM, Willy-Brandt-Platz \\
\hline 16 & $\mathrm{~m}$ & 35 & deutsch & Drucker arbeitssuchend & unter $500 €$ & S-Bahn & Mainz-Kastel \\
\hline 17 & w & 19 & deutsch & $\begin{array}{ll}-- \\
\end{array}$ & unter $500 €$ & S-Bahn & Kelsterbach \\
\hline 18 & w & 25 & deutsch & Personaldienstleisterin & 2.000 bis unter $3.000 €$ & S-Bahn & Bad Soden \\
\hline 19 & $\mathrm{~m}$ & 21 & sudanesisch & KfZ-Mechaniker & unter $500 €$ & Bus & Bad Schwalbach, Adolfstraße \\
\hline 20 & $\mathrm{~m}$ & 18 & deutsch & BvB - Gesundheit \& Soziales & unter $500 €$ & Bus & Wiesbaden, Elsässer Platz \\
\hline 21 & $w$ & 49 & deutsch & Arbeitsvermittlerin & 2.000 bis unter $3.000 €$ & U-Bahn, Stationskontrolle & FFM, Leipziger Straße \\
\hline 22 & $\mathrm{~m}$ & 40 & deutsch & Transport \& Logistik Ausbildung & unter $500 €$ & U-Bahn, Stationskontrolle & FFM, Leipziger Straße \\
\hline 23 & w & 28 & rumänisch & Produktionshelferin & 1.000 bis unter $2.000 €$ & U-Bahn, Stationskontrolle & FFM, Leipziger Straße \\
\hline 24 & $\mathrm{~m}$ & 16 & türkisch & Schüler & unter $500 €$ & S-Bahn & FFM, Hauptwache \\
\hline 25 & w & 26 & deutsch & IT-Systemfachfrau & 1.000 bis unter $2.000 €$ & S-Bahn & FFM, Griesheim \\
\hline 26 & w & 19 & deutsch & Verkäuferin & 1.000 bis unter $2.000 €$ & S-Bahn & FFM, Hauptbahnhof \\
\hline 27 & w & 26 & deutsch & Journalistin & 2.000 bis unter $3.000 €$ & U-Bahn, Stationskontrolle & FFM, Grüneburgweg \\
\hline 28 & w & 22 & US-amerikanisch & Erzieherin & 500 bis unter $1.000 €$ & U-Bahn, Stationskontrolle & FFM, Grüneburgweg \\
\hline 29 & w & 47 & serbisch & Pflegedienst & 500 bis unter $1.000 €$ & U-Bahn, Stationskontrolle & FFM, Grüneburgweg \\
\hline 30 & $\mathrm{~m}$ & 33 & deutsch & Wissenschaftlicher Mitarbeiter & 1.000 bis unter $2.000 €$ & U-Bahn, Stationskontrolle & FFM, Grüneburgweg \\
\hline 31 & $\mathrm{~m}$ & 45 & deutsch & Drucker & 1.000 bis unter $2.000 €$ & U-Bahn, Stationskontrolle & FFM, Kirchplatz \\
\hline
\end{tabular}




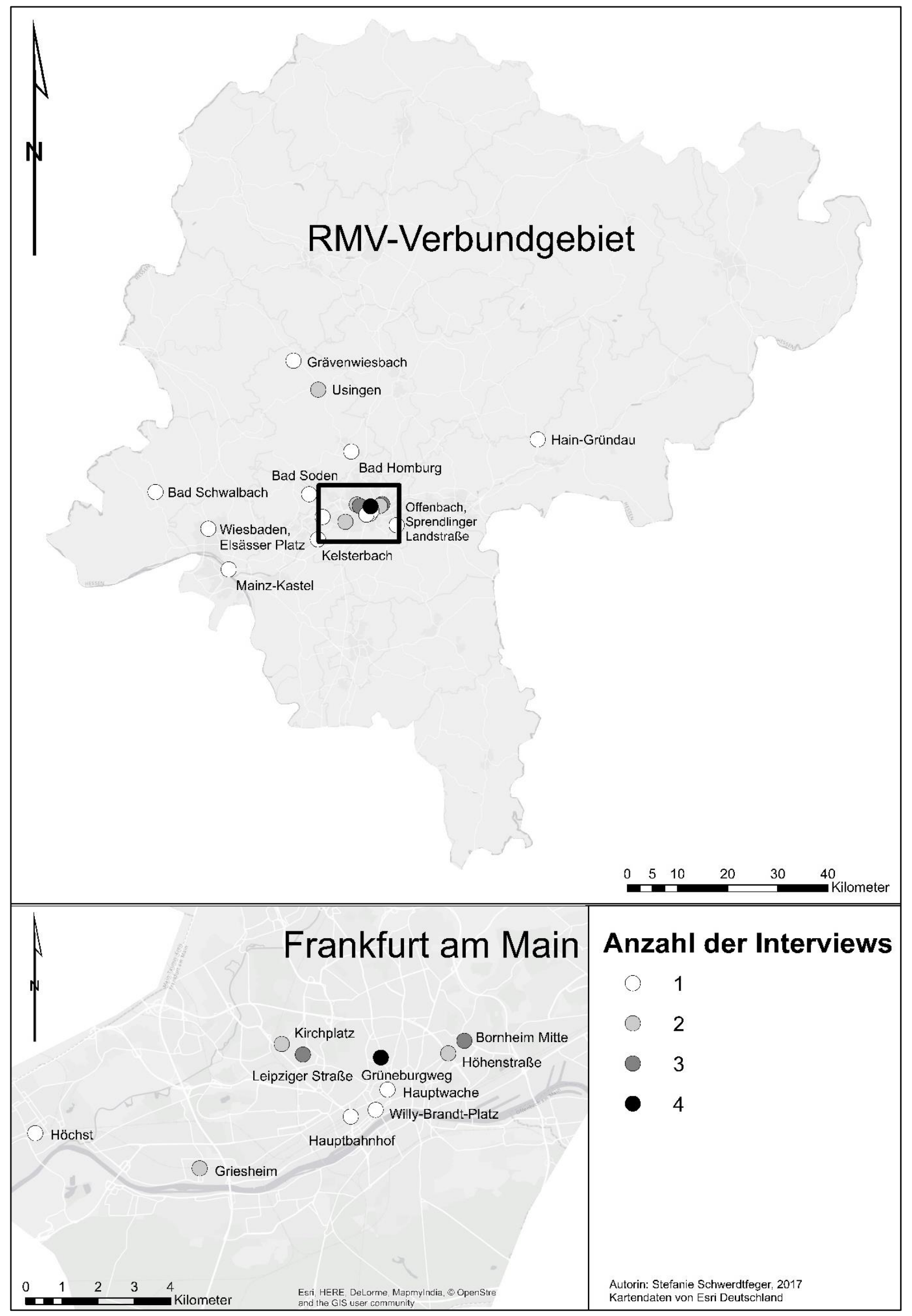

Abbildung 2: Übersicht der Befragungsorte im RMV-Verbundgebiet Quelle: eigene Darstellung 


\subsubsection{Sicherung des empirischen Datenmaterials}

Die Interviews wurden digital aufgezeichnet. Dies ermöglicht die gesamte Erfassung des gesprochenen Interviews und dient als Basis für eine Transkription, welche schließlich eine Grundlage für die wissenschaftliche Auswertung schafft (Witzel 1982: 91). Außerdem wurden bereits während des Gespräches Notizen, Bemerkungen und erste Deutungshinweise im Sinne von Memos im ausgedruckten Interviewleitfaden vermerkt. Direkt nach dem Gespräch wurde eine kurze Zusammenfassung des Interviews verfasst, die darüber hinaus die Entstehungssituation des Gespräches sowie den eventuellen Nachgang an ein Gespräch dokumentiert.

Zur Vorbereitung der rekonstruktiven Auswertung der problemzentrierten Interviews mit der dokumentarischen Methode (Kapitel 2.3) wurden die aufgezeichneten Interviews vollständig transkribiert. Dabei wurde das Transkriptionssystem TiQ - Talk in Qualitative Social Research angewendet (Bohnsack 2003: 235; Przyborski \& Wohlrab-Sahr 2008: 164 ff.). Der Vorteil gegenüber anderen Transkriptionssystemen ist die Lesbarkeit für die Auswertung mit der dokumentarischen Methode.

\subsection{Auswertung: Dokumentarische Methode}

Die Interviews wurden mithilfe der dokumentarischen Methode ausgewertet. Die dokumentarische Methode ist eine Auswertungsmethode für offene und narrative Interviews, Gruppendiskussionen, historische Texte sowie für Bilder, Fotos oder Videos und wird vorranging in den Sozial- und Erziehungswissenschaften angewendet. Sie steht in der Tradition der Wissenssoziologie Karl Mannheims und hat somit einen ethnomethodologischen Hintergrund. Die dokumentarische Methode bietet ein Analyseverfahren an, welches einen Zugang sowohl zum reflexiven als auch zum handlungsleitenden Wissen der Akteurlnnen und somit zur Handlungspraxis liefert. Das Ziel ist es, diese Handlungspraxis zu rekonstruieren. Dabei tritt das der Praxis zugrundeliegende habitualisierte und teilweise inkorporierte Orientierungswissen in den Vordergrund (Bohnsack et al. 2013: 9).

Das übergeordnete Ziel der dokumentarischen Interpretation ist die Rekonstruktion des Zusammenhanges zwischen Erfahrungen und Orientierungen, die in qualitativen Erhebungsmethoden vermittelt werden (Bohnsack 2003: 33 ff.). Die Forschungspraxis der dokumentarischen Methode lässt sich in drei Arbeitsschritte einteilen: (1) die formulierende Interpretation, (2) die reflektierende Interpretation und (3) die Typenbildung. Bereits vor diesen drei Auswertungsschritten werden thematische Verläufe des auszuwertenden Materials erstellt, anhand derer die zu interpretierenden Passagen ausgewählt und transkribiert werden. Die Auswahl der auszuwertenden Passagen erfolgt anhand formaler (Fokussierungsmetaphern, Pausen, Textsortenwechsel) sowie inhaltlicher Gesichtspunkte in Form jener Themen, die für das Erkenntnisinteresse von Bedeutung sind (Przyborski 2004: 50 ff.). 


\section{Motive des Fahrens ohne (gültigen) Fahrschein}

In diesem Abschnitt werden die Motive des Fahrens ohne (gültigen) Fahrschein in Form einer Typologie vorgestellt und beschrieben. Die Typologie ist das Ergebnis eines explorativen Forschungsdesigns der Beweggründe des Fahrens ohne (gültigen) Fahrschein. Die Auswertung hat zunächst eine Unterscheidung zwischen intentionalem und nicht-intentionalem Handeln ergeben. Intentionen stellen nach Delbosc und Currie (2016) ein bedeutendes Kriterium bei der Segmentierung des Fahrens ohne (gültigen) Fahrschein dar. Demnach können Personen, die in der Regel für die Fahrt mit ÖPNV bezahlen, dem nicht-intentionalen Fahren ohne (gültigen) Fahrschein zugeordnet werden. Die Personen, die den ÖPNV regelmäßig ohne (gültiges) Ticket benutzen werden hingegen in den Typen des intentionalen Fahrens ohne (gültigen) Fahrschein beschrieben. Unter dieser ersten Ebene enthält die Typologie sechs unterschiedliche Typen (Abbildung 4).

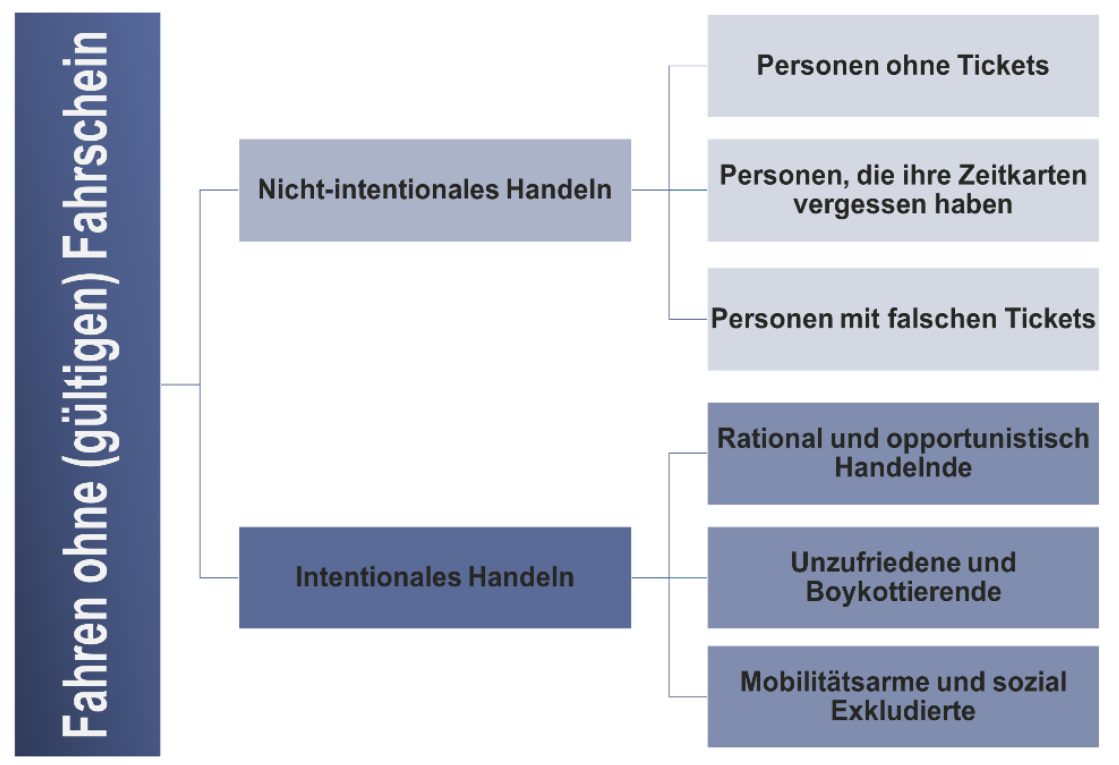

Abbildung 3: Typologie des Fahrens ohne (gültigen) Fahrschein Quelle: eigene Darstellung

Neben der Intention zum Fahren ohne (gültigen) Fahrschein konnten sechs weitere Merkmale analysiert werden, anhand derer die Typen des Fahrens ohne (gültigen) Fahrschein verglichen werden können und die bei der Zuordnung der Fälle zu den Typen eine wichtige Rolle spielten: (1) Akzeptanz des Fahrens ohne (gültigen) Fahrschein, (2) Akzeptanz des Straftatbestandes, (3) Normkonformität, (4) Risikobereitschaft, (5) Fahren ohne (gültigen) Fahrschein im sozialen Umfeld, und (6) Fahren ohne (gültigen) Fahrschein als Geheimnis. Die Zusammensetzung der Typologie aus den einzelnen Fällen wird in Tabelle 2 dargestellt:

Tabelle 2: Zusammensetzung der Typologie des Fahrens ohne (gültigen) Fahrschein (eigene Darstellung)

\begin{tabular}{|c|l|l|}
\hline \multicolumn{2}{|c|}{ Motivtyp } & Fälle (Interviewnummer) \\
\hline \multirow{2}{*}{$\begin{array}{c}\text { nicht- } \\
\text { intentionales } \\
\text { Handeln }\end{array}$} & Personen ohne Tickets & $2,4,15,17,26,27,31$ \\
\cline { 2 - 3 } & Personen, die ihre Zeitkarten vergessen haben & $3,6,8,25,29$ \\
\cline { 2 - 3 } & Personen mit falschen Tickets & $18,20,21$ \\
\hline \multirow{2}{*}{$\begin{array}{c}\text { intentionales } \\
\text { Handeln }\end{array}$} & rational und opportunistisch Handelnde & $1,10,23,24,28 *$ \\
\cline { 2 - 3 } & Unzufriedene und Boykottierende & $7,11,12,13,14,22,30^{*}$ \\
\cline { 2 - 3 } & Mobilitätsarme und sozial Exkludierte & $5,9,16,19$ \\
\hline *Auslöser des Fahrens ohne (gültigen) Fahrschein: Armutserfahrungen \\
\hline
\end{tabular}




\subsection{Nicht-intentionales Fahren ohne (gültigen) Fahrschein}

Nicht-intentionales Fahren ohne (gültigen) Fahrschein ist im Rahmen der qualitativen Interviews bei drei unterschiedlichen Typen aufgetreten: Bei (1) Personen ohne Tickets, (2) Personen, die ihre Zeitkarten vergessen haben und bei (3) Personen mit falschen Tickets. Eine Beschreibung der jeweiligen Typen ist in den folgenden Unterkapiteln enthalten.

\subsubsection{Motivtyp: Personen ohne Tickets}

Der Typ Personen ohne Tickets besteht aus BezieherInnen von Kurzstreckentickets, Einzelfahrscheinen, Tagestickets oder Zeitkarten mit kurzer Laufzeit, wie Wochenfahrkarten, die aufgrund besonderer Umstände ohne Ticket kontrolliert wurden. Es handelt sich um Personen, die den ÖPNV entweder nur selten oder unregelmäßig benutzen, oder aber um tägliche NutzerInnen, für die sich eine Dauerzeitkarte - aus unterschiedlichen Gründen - finanziell nicht rechnet. Die Motive des Fahrens ohne (gültigen) Fahrschein werden mit der Schilderung besonderer Umstände begründet. Beispiele dafür sind einerseits monetäre Aspekte, zum Beispiel das Vergessen des Portemonnaies oder das Nichtvorhandensein von passendem Kleingeld am Fahrkartenautomat:

"Und [...] ich habe nur zwanzig Euro. Aber [...] [der] nimmt es nicht. Dann habe ich versucht, mit meiner Bankkarte, hab so [eine] niederländische Bankkarte, und [die] nimmt er auch nicht. Ich habe versucht. Jedes Mal zurückgegeben. Und dann habe ich gedacht: Okay, dann fahr ich doch, ich muss [zu] meiner Arbeit."

Interview 15, männlich, 57 Jahre

Andererseits spielen auch zeitliche Aspekte eine Rolle, insbesondere bei geringen Taktdichten gekoppelt mit Eile oder schlechtem Wetter:

„ICh bin in der Hauptwache eingestiegen und hier wieder ausgestiegen, das sind zwei Stationen. Ich laufe das eigentlich immer. Und heute dachte ich: Scheiße, ich bin spät dran, es regnet, eingestiegen. Ah ja, ehrlich gesagt, schon wissend, dass das nicht legal ist."

Interview 27, weiblich, 26 Jahre

In den zwei Zitaten wird vor allem der zweckrationale Aspekt des Fahrens ohne (gültigen) Fahrschein sichtbar. In beiden Beispielen ist den Befragten bewusst, dass sie als Fahrgast dazu verpflichtet sind, vor Antritt der Fahrt ein Ticket für den ÖPNV zu lösen. Obwohl sie dieser Verpflichtung in der Regel nachkommen, entscheiden sie sich aufgrund zweckrationaler Beweggründe für das Fahren ohne Fahrschein. Ein ausschlaggebender Grund für die Entscheidung ist dabei die wahrgenommene Prüfquote. Fahrkartenkontrollen werden entweder gar nicht bis selten oder aber zu anderen Tageszeiten wahrgenommen, was die Kalkulation des eingegangenen Risikos beeinflusst:

„Ja, ich habe mir gedacht, ich schlupf jetzt mal mit den Schülern durch und ich hoffe, jemand drückt ein Auge zu für mich, ja. Aber dem war wohl nicht so. Ich bin ein bisschen überrascht, habe jetzt gedacht, die versteckte Kamera kommt raus, ja. Weil es ist echt, ja, unglaublich, ja. Ich wurde noch nie kontrolliert und dann ausgerechnet heute." 
Einstellungen und Meinungen über das Fahren ohne (gültigen) Fahrschein haben bei den Personen ohne Tickets einen ablehnenden Charakter: Kein/e Befragte/r akzeptiert die Entscheidung, kein Ticket für den ÖPNV zu kaufen, oder bewertet es als moralisch vertretbar. Aus diesem Grund werden die Erteilung von erhöhten Beförderungsentgelten und die Einstufung als Straftat akzeptiert; letzteres vor allem bei wiederholtem Fahren ohne (gültigen) Fahrschein.

Eine Intention zum Fahren ohne (gültigen) Fahrschein ist bei diesem Typus nicht vorhanden. Die Hintergründe des Fahrens ohne (gültigen) Fahrschein beziehen sich vor allem auf den Fahrkartenerwerb: Eile oder kein passendes Zahlungsmittel bereit zu haben führen dazu, dass sich Personen - vor dem Hintergrund der Erreichung ihres Ziels - mehr oder minder dazu genötigt sehen, die Fahrt mit den öffentlichen Verkehrsmittel ohne Fahrschein anzutreten. Zusammenfassend lässt sich der Motivtyp mit einer hohen Normkonformität in Bezug auf den Ticketkauf sowie einer hohen Akzeptanz des Straftatbestandes beschreiben. Die Risikobereitschaft ist eher gering und ist durch die subjektive Wahrnehmung von Fahrkartenkontrollen bedingt. Im sozialen Umfeld wird in der Regel nicht ohne (gültigen) Fahrschein gefahren: Es passiert aus Versehen und kommt nicht häufig vor. Über das eigene Fahren ohne (gültigen) Fahrschein wird anekdotisch mit FreundInnen und Familie gesprochen. Die Intention zum Fahren ohne (gültigen) Fahrschein ist nur geringfügig ausgeprägt, da diese Handlung moralisch abgelehnt wird.

\title{
3.1.2 Motivtyp: Personen, die ihre Zeitkarten vergessen haben
}

Neben den Personen, die lediglich aufgrund besonderer Umstände nicht für die Fahrt mit öffentlichen Verkehrsmitteln zahlen, hat sich in der Kategorie der nicht-intentionalen KundInnen der Typus der Personen, die ihre Zeitkarten vergessen haben, herausgebildet. Dabei handelt es sich um KundInnen von Zeitkarten, Studierende, die über ein Semesterticket verfügen, sowie um BezieherInnen eines JobTickets oder der CleverCard, die diese entweder zu Hause vergessen haben oder die den Gültigkeitszeitraum nicht beachtet und in der Folge versäumt haben, die Zeitkarte o.ä. zu verlängern oder neu zu validieren:

\begin{abstract}
"Ich hatte wohl meinen Studentenausweis nicht in meiner Geldbörse dabeigehabt und dementsprechend... Ich wollte Einkaufen fahren, gerade, und normalerweise habe ich den Studentenausweis für die derartigen Fahrtwege. Und dummerweise habe ich den im Handy oder in einem dieser Fächer oder in der Geldbörse oder vermutlich zuhause liegen lassen."
\end{abstract}

Interview 6, männlich, 22 Jahre

Der Typus zeichnet sich durch eine hohe Zahlungsbereitschaft aus, sowohl für die vergünstigten Zeitkartenmodelle, als auch für höherpreisige Dauerkarten. Analog zum Typus Personen ohne Tickets haben die Einstellungen und Meinungen über das Fahren ohne (gültigen) Fahrschein einen ablehnenden Charakter. Daher wird die Einstufung als Straftat von Personen, die ihre Zeitkarten vergessen haben, als fair und nachvollziehbar beschrieben: 
„[...] weil es ja nicht nur bezogen ist auf Verkehrsunternehmen. Also grundsätzlich, dass jemand sich irgendwie Leistungen erschleicht, warum sollte der nicht bestraft werden? Während jemand, der irgendwie ein Brötchen mitgehen lässt, im Supermarkt, bestraft wird. Also, dass da ein anderes Vermögen geschadet oder Vermögen oder Eigentum beschädigt wird, finde ich völlig berechtigt, dass das beides pönalisiert wird. Letztlich, gut, die Strafe, die einen im Zweifel ja mehr interessiert ist erstmal die monetäre, weil das ja [...] strafrechtlich verfolgt wird. [Da] dauert es zumindest drei, vier, fünf Mal, die man irgendwie erst gespeichert werden muss, glaube ich, bevor man mit der Polizei zu tun hat."

Interview 8, weiblich 27 Jahre

Eine Intention zum Fahren ohne Fahrschein besteht bei dem Typ Personen, die ihre Zeitkarten vergessen haben, nicht, sondern es handelt sich um ein Versehen. Dies wird auch in der Wahrnehmung der Situation sichtbar, da sich die Personen nicht als jene sehen, die Dienstleistungen erschleichen, sondern als zahlende KundInnen:

„Es gehört sich einfach. Das versucht man sich schon einzuprägen. Wie gesagt, das ist kein Ding, einfach mal eine Fahrkarte zu ziehen. Aber ich bin eh außen vor, da ich den Studentenausweis habe."

Interview 6, männlich, 22 Jahre

Der Motivtyp Personen, die ihre Zeitkarten vergessen haben, zeichnet sich insbesondere durch eine niedrige Risikobereitschaft aus, da es sich um zahlende KundInnen von Zeitkarten handelt. Eine Intention zum Fahren ohne (gültigen) Fahrschein ist nicht erkennbar, da es moralisch abgelehnt wird. Im sozialen Umfeld kommt das Fahren ohne (gültigen) Fahrschein als Ausnahme vor, in der Regel wird aber nicht darüber gesprochen, da der Ticketerwerb für eine Fahrt mit dem ÖPNV als selbstverständlich angesehen wird. In diesem Motivtyp herrscht eine hohe Normkonformität und die Einstufung des Fahrens ohne (gültigen) Fahrschein wird ausnahmslos akzeptiert. Schließlich verfügen die Personen, die dem Typ zugeordnet wurden über eine Fahrkarte, die lediglich vergessen wurde.

\subsubsection{Motivtyp: Personen mit falschen Tickets}

Ein dritter Typus, Personen mit falschen Tickets, bei dem keine Intention zum Fahren ohne (gültigen) Fahrschein erkennbar ist, bezieht sich auf Informationsdefizite. Im Gegensatz zu den bereits beschriebenen Typen weist er eine starke Heterogenität auf, was bedeutet, dass Informationsdefizite unterschiedlicher Art zum Fahren ohne gültigen Fahrschein führen können. Aus dem Interviewmaterial konnten zwei unterschiedliche Arten herausgelesen werden. Informationsdefizite in Bezug auf: (1) Tarifgebiete sowie -grenzen und (2) den Umgang mit Zeitkarten.

Informationsdefizite in Bezug auf Tarifgebiete sowie -grenzen führen vor allem bei KundInnen von Kombitickets und bei TouristInnen zum Fahren ohne gültigen Fahrschein: In der Regel verfügen die Personen über ein Ticket, welches allerdings in dem kontrollierten Tarifgebiet oder zum Kontrollzeitpunkt nicht (mehr) gültig ist. Während der Befragung stellte sich heraus, dass Unklarheiten in Bezug auf die Gültigkeit von Kombinationsangeboten bestehen: 
"ICh hatte ein ICE-Ticket, das mir der Arbeitgeber gezahlt hat. Und ich bin davon ausgegangen, weil da ja ,mit City' draufsteht - also ich habe das jetzt auch noch nicht so oft gemacht - ich dachte einfach, da ist dann quasi das RMV-Ticket mit drinnen. [...] Dass einfach die Nahverkehrsfahrt, die man quasi im Anschluss macht, mit dran ist. Ich dachte jetzt nicht, dass die an ein Tarifgebiet gebunden ist, oder so."

Interview 18, weiblich, 25 Jahre

Die fehlende Intention verdeutlicht vor allem die moralische Ablehnung des Fahrens ohne gültigen Fahrschein. Die befragten Personen verstehen sich nicht als jene, die kein Ticket für die Fahrt mit den öffentlichen Verkehrsmitteln kaufen und bewerten das Fahren ohne (gültigen) Fahrschein als unmoralisch:

„[...] wir haben uns nicht als Schwarzfahrer gesehen. [...] Das ist ja auch nicht gut. Ich würde ja auch immer eine Karte kaufen, ja. Das soll man ja auch nicht. Das will ich damit ja nicht irgendwie schönreden.

Interview 21, weiblich, 49 Jahre

Informationsdefizite in Bezug auf den Umgang mit Zeitkarten können in einigen Fällen auch zur Verärgerung bereits zahlender KundInnen führen. Aus den Interviews sind diesbezüglich zwei Beispiele hervorgegangen. In einem ersten Beispiel wurde eine Bezieherin von Monatskarten nicht über die Möglichkeit der Personalisierung der Monatskarte informiert. In einem zweiten Beispiel war eine falsche Kundlnnennnummer auf einer CleverCard eingetragen. Außerdem war der Bezieher der CleverCard nicht über den Gültigkeitszeitraum seiner Fahrkarte in Kenntnis gesetzt worden, er konnte zwar bei einer Fahrkartenkontrolle ein Ticket vorweisen, dieses war allerdings ungültig:

"Das wusste ich nicht, weil [...] ich dachte, wenn da irgendwas nicht stimmt, dann spricht mich der Busfahrer darauf an. Also von daher habe ich mir auch keine Gedanken gemacht oder gar nichts. Hätte ich es gewusst, hätte ich natürlich eine neue geholt."

Interview 20, männlich, 18 Jahre

Die geschilderten Beispiele veranschaulichen wie Informationsdefizite vor allem bei zahlenden KundInnen zum Fahren ohne gültigen Fahrschein führen können. Sie lehnen entsprechendes Verhalten moralisch ab und ärgern sich über die Ausstellung eines erhöhten Beförderungsentgeltes. Sie wünschen sich mehr Professionalität, Informationen oder Kulanz seitens der Verkehrsunternehmen.

Zusammenfassend lässt sich der Motivtyp Personen mit falschen Tickets mit einer geringen Akzeptanz für das Fahren ohne (gültigen) Fahrschein beschreiben, eine Intention zur entgeltfreien ÖPNV-Nutzung ist nicht erkennbar. Im sozialen Umfeld kommt das Fahren ohne (gültigen) Fahrschein aus Versehen vor und es wird anekdotisch darüber gesprochen. Die Risikobereitschaft ist relativ gering, da es sich um zahlende KundInnen handelt, die nicht ausreichend über die Gültigkeit ihrer Fahrkarte informiert sind. Daher charakterisiert diesen Motivtyp auch eine hohe Normkonformität. Der Straftatbestand wird größtenteils akzeptiert, allerdings wird das eigene Fahren ohne gültigen Fahrschein teilweise nicht als solches angesehen. 


\subsection{Intentionales Fahren ohne (gültigen) Fahrschein}

Die Personengruppe derjenigen, die nicht für ihre Fahrt mit den öffentlichen Verkehrsmitteln zahlen, ist in der Gesamttypologie mit drei Typen vertreten: (1) rational und opportunistisch Handelnde, (2) Unzufriedene und Boykottierende und (3) Mobilitätsarme und sozial Exkludierte.

\subsubsection{Motivtyp: Rational und opportunistisch Handelnde}

Dem Typ rational und opportunistisch Handelnde wurden Personen zugeordnet, die den ÖPNV grundsätzlich ohne Ticket benutzen. Die Beweggründe beziehen sich dabei auf finanzielle Ersparnisse. Auslöser für die Fahrt ohne Fahrschein ist die Wahrnehmung einer niedrigen bis hin zu gar keiner Prüfquote auf der genutzten ÖPNV-Verbindung. Dies führt schließlich zu der Entscheidung, den ÖPNV künftig ohne Ticket zu benutzen:

"Ich hatte einfach keine Fahrkarte. Ich wohne in Deutschland seit zwei Jahren und im ersten Jahr hatte ich eine Monatsdauerkarte gekauft. Und ich habe gemerkt, dass es keine Kontrolle gibt. Und ein paar Freunde haben mir [...] erklärt: Okay, ich habe keine Karte, ja. Und deswegen habe ich das abgesagt. Ich habe keine Monatsdauerkarte. Ja, ich habe einfach keine Karte. Und es war eigentlich das erste Mal, dass jemand mich kontrolliert hat."

Interview 1, männlich, 28 Jahre

Der primäre Beweggrund ist nicht die Ablehnung von Tarifen. Vielmehr ergründet sich die Fahrt ohne Fahrschein auf eine individuelle, rationale Abwägung des Risikos in eine Kontrolle zu kommen mit dem finanziellen Gewinn. In dem Typ sind auch Personen vertreten, die sich in finanziell prekären Situationen befinden. Dabei handelt es sich u.a. um in Aus- oder Weiterbildungssituationen befindliche Personen:

"ICh bin Schwarzfahrer, seitdem ich die Ausbildung hab. Und das ist jetzt das dritte Mal in diesen zweieinhalb Jahren, dass ich erwischt wurde. Deswegen. Ich krieg eigentlich die Fahrkarte bezahlt vom Arbeitsamt aber, hm, ja. [...] Ich geh sie halt nicht kaufen."

Interview 10, männlich, 28 Jahre

Das Fahren ohne Fahrschein wird als gerechtfertigt oder gar als logische Konsequenz beschrieben. Dies kann sich sowohl auf Unzufriedenheit mit Service-Aspekten wie Sauberkeit oder Pünktlichkeit, aber auch auf Fahrkartenkontrollen beziehen: "Aber ich mein, wir sind junge Leute und irgendwie müssen wir auch hier
angespornt werden uns hier eine Fahrkarte zu holen, so ein bisschen".

Interview 28, weiblich, 22 Jahre

Bei diesem Typ besteht eine starke Intention zum Fahren ohne Fahrschein. Es handelt sich um Vielnutzende des ÖPNV, die bewusst nicht für die Fahrt mit öffentlichen Verkehrsmitteln bezahlen und ihr Handeln als logische Konsequenz auf die Wahrnehmung einer niedrigen Prüfquote sehen. Sie rechtfertigen ihr Verhalten etwa mit mangelndem Service, eine moralische Ablehnung des Fahrens ohne Fahrschein lässt sich nicht erkennen. Vielmehr steht der finanzielle Gewinn, der sich aus der entgeltfreien ÖPNV-Nutzung ergibt, im Mittelpunkt des Motivs. Im sozialen Umfeld kommt das Fahren ohne (gültigen) Fahrschein häufig vor und es wird oft darüber gesprochen, jedoch mit Ausnahme: Das 
Fahren ohne Fahrschein kann gleichermaßen als private Entscheidung, über die mit Freundlnnen und Familie nicht gesprochen wird, gelten. Die Normkonformität ist innerhalb des Motivtyps nur gering ausgeprägt. Zudem wird der Straftatbestand des Fahrens ohne Fahrschein nicht nachvollzogen und teilweise belächelt.

\subsubsection{Motivtyp: Unzufriedene und Boykottierende}

Beim Typ Unzufriedene und Boykottierende stehen allgemein Ablehnungsmotive im Vordergrund. Prominent ist dabei die Ablehnung der Preise für die Nahverkehrsfahrt:

„[...] Und wollte jetzt auf die Arbeit und normalerweise mach ich das tatsächlich meistens mit dem Rad oder zu Fuß und wenn ich mit der Bahn fahre, dann zahl ich tatsächlich nur jedes zweite Mal, (lachend) weil ich das unverschämt finde, die Preise."

Interview 12, weiblich, 32 Jahre

Neben der Ablehnung von Preisen kommen bei diesem Typ auch vermehrt politische Motive zum Tragen:

„ICh denke, Verkehr sollte öffentlich sein und sollte auch jedem zugänglich sein. Dann hätten wir auch weniger Autos auf der Straße. Und das ist alles mal bezahlt worden, von unseren Eltern und [...] man hat es dann irgendwann in eine Aktiengesellschaft umgewandelt. Ja, und jetzt müssen die Dividenden laufen und jetzt erhöht man jedes Mal die Preise, ne? Das ist einfach mit nichts mehr gerechtfertigt, ja?"

Interview 22, männlich, 40 Jahre

Bei den Ablehnungsmotiven handelt es sich um persönliche Wahrnehmungen, Meinungen und Einstellungen gegenüber tariflichen Bestimmungen und verkehrs- sowie sozialpolitischen Entscheidungen. Die Wahrnehmung einer niedrigen Prüfquote kann dabei als handlungsverstärkend interpretiert werden:

„Ja, warum habe ich keine Fahrkarte? Ich sehe das halt nicht ein, das ist viel zu teuer mit der Fahrkarte und bis jetzt wurde ich auch nie kontrolliert hier. Und deshalb habe ich das auch gemacht. Weil ich sehe es halt nicht ein. Weil Du bezahlst, selbst wenn Du nach Frankfurt fährst, bezahlst Du fast fünf Euro. Echt, da kann man auch laufen, eigentlich."

Interview 14, männlich, 25 Jahre

Analog zum Typ rational und opportunistisch Handelnde wird das Fahren ohne Fahrschein als gerechtfertigt oder gar als logische Konsequenz angesehen, allerdings nicht aufgrund der Wahrnehmung einer niedrigen Prüfquote, sondern aufgrund von Einstellungen und Meinungen zu den Preisen der ÖPNV-Nutzung. Verkehrsunternehmen oder -verbünde werden nicht als Erbringer einer öffentlichen Dienstleistung angesehen, sondern als privatwirtschaftliche Unternehmen, denen es um Gewinnmaximierung geht: 
„Naja, eigentlich sollte echt jeder schwarzfahren, damit die mal merken, was Sache ist. Ja, weil in Spanien ist das ja auch so, ja? Die Securities, die kontrollieren gar keine Fahrkarte mehr, ja? Nur noch, dass da kein Scheiß mehr passiert in den Bahnen. Ja, einfach, weil die der Sache nicht mehr Herr werden. Es kann sich halt kein Mensch mehr leisten, die fahren einfach schwarz, die Gerichte kommen nicht mehr hinterher und fertig ist. Man kann das schon aushebeln."

Interview 22, männlich, 40 Jahre

Im Kontext dieser Argumentationslogik wird das Fahren ohne Fahrschein zudem nicht als Straftat verstanden:

"Allein die Gerichte damit zu belasten. Das ist, ey! Das ist wegen Schwarzfahren. Die sperren die Leute ins Gefängnis deswegen. Die Leute werden eingesperrt. [...]"

Interview 13, männlich, 48 Jahre

"Ne, ich find Gefängnisstrafen lächerlich. [...] Auch für eine Dienstleistung, die hier sozusagen erbracht wird. Das ist nichts Abgesperrtes, [...] Privates."

Interview 7, männlich, 21 Jahre

Auch bei diesem Typ besteht eine grundsätzliche Intention zum Fahren ohne Fahrschein. Es handelt sich um Vielnutzende des ÖPNV, die bewusst nicht für die Fahrt mit öffentlichen Verkehrsmitteln bezahlen und ihr Handeln als Konsequenz auf ihre Wahrnehmung der Tarifgestaltung sehen. Die Risikobereitschaft ist bei diesem Motivtyp stark ausgeprägt und das Fahren ohne (gültigen) Fahrschein wird nicht als deviante Verhaltensweise angesehen, was sich durch eine sehr geringe Normkonformität bedingt. Zudem wird das Fahren ohne Fahrschein nicht als Straftat, sondern eher als Bagatelle interpretiert. Im sozialen Umfeld kommt das Fahren ohne (gültigen) Fahrschein teilweise vor, weswegen es auch nicht als geheime Handlung beschrieben wird. Vielmehr wird die eigene Entscheidung, den ÖPNV ohne (gültiges) Ticket zu benutzen, aufgrund der Wahrnehmung der Tarifgestaltung, verteidigt.

\subsubsection{Motivtyp: Mobilitätsarme und sozial Exkludierte}

Dem Typ Mobilitätsarmut und sozial Exkludierte wurden Personen zugeordnet, die den ÖPNV bewusst ohne Fahrschein benutzen, diese Handlung allerdings als Zwang beschreiben. Auslöser für den beschriebenen Zwang sind dabei finanzieller Art: Es handelt sich um Personen in Umbruchphasen und Personen, die Bildungsabschlüsse nachholen, aber auch um Personen ohne festen Wohnsitz. Die Motive des Typs Mobilitätsarme und sozial Exkludierte, die sich aus der finanziellen Situation ergeben, sind allerdings nicht mit denen des Typs rational und opportunistisch Handelnde gleichzusetzen: Sie entsprechen zwar einer nutzenmaximierenden Handlungslogik, unterliegen allerdings Zwängen, die sich negativ auf das Sozialkapital der betroffenen Personen auswirken können: 
„ICh kam gerade von der Tafel [...], weil ich ja dann mit drei Tüten bin und das ziemliche schwer ist, wollte ich halt jetzt nicht zu Fuß kommen [...]. Deswegen bin ich halt zwei Stationen gefahren. Das mach ich eigentlich öfter so und ich hatte keine Fahrkarte, weil ich kein Geld hatte. Hätte ich Geld gehabt, hätte ich mir eine geholt. [...] Ich hatte keine Wahl, also ich hätte natürlich laufen können."

„ICh treffe mich jetzt auch nicht so oft mit Freunden [...], dann muss ich wieder mit der Bahn fahren."

Interview 9, weiblich, 22 Jahre

Im ersten Teil des Zitats aus Interview 9 wird vor allem die finanziell prekäre Situation aber auch der Wunsch, eine Fahrkarte erwerben zu können, erkennbar. Im zweiten Teil werden die möglichen negativen Auswirkungen auf das Sozialkapital sichtbar. Besonders der Wunsch oder Wille, eine Fahrkarte für den ÖPNV erwerben zu können, ist eine wichtige Eigenschaft dieses Typs:

„Wenn wir Schüler [wären], dann können wir einfach [ein] Angebot für die Fahrkarte [bekommen]. Ich war schon da und ich habe gefragt: 'Wie viel kostet [die] Monatsfahrkarte von Bad Schwalbach nach Taunusstein?' Sie haben mir gesagt: 'Ungefähr sechsundsechzig Euro.' So, das konnte ich einfach nicht [bezahlen]."

Interview 191, männlich, 21 Jahre

Der Straftatbestand des Fahrens ohne (gültigen) Fahrschein wird nachvollzogen; im selben Zuge wird aber auch der Umgang der Verkehrsunternehmen sowie der Kommunalpolitik mit dem Problem kritisiert:

\begin{abstract}
"Man kann eine Haftstrafe davon bekommen, ja, gut. Muss man in Kauf nehmen, das ist halt der Preis vom Leben. [...] Aber ich sag mal so: Andere kriegen ja auch irgendwie was, wo sie günstiger mit fahren können. Rentner, Studenten, bla, bla, bla. Und ich weiß nicht, warum man bei Obdachlosen nicht auch sowas machen kann, einfach so was wie einen Obdachlosenpass oder so [...], wo man sagt: 'Okay, der Obdachlose kann jetzt von hier nach da fahren, weil er sich ja eigentlich nur das holt, was ihm eigentlich für diesen Tag zusteht².' Er fährt ja nicht mit der Bahn, weil er jetzt Spaß hat - oh, ich guck mir gern die Gegend an."
\end{abstract}

Interview 16, männlich, 35 Jahre

Der Typ Mobilitätsarme und sozial Exkludierte zeichnet sich dadurch aus, dass die Personen den Wunsch äußern, ein Ticket für den ÖPNV erwerben zu können. Jedoch kann diesem Wunsch aufgrund fehlender finanzieller Ressourcen nicht nachgegangen werden. Eine Intention zum Fahren ohne (gültigen) Fahrschein ist dennoch vorhanden, da es sich um eine bewusste Entscheidung handelt, den ÖPNV ohne (gültiges) Ticket zu benutzen. Der Straftatbestand des Fahrens ohne (gültigen) Fahrschein wird akzeptiert und nachvollzogen, der Umgang mit daraus resultierenden Tendenzen zu Mobilitätsarmut und potenzieller sozialer Exklusion wird jedoch kritisiert. Das Fahren ohne (gültigen) Fahrschein wird moralisch abgelehnt, was die Wahrnehmung als finanziell bedingten Zwang verstärkt. Die Risikobereitschaft innerhalb des Motivtyps weder hoch noch niedrig und variiert je nach Situation.

\footnotetext{
${ }^{1}$ Grund für Fahrt ohne (gültigen) Fahrschein: Es wurde ein Gruppenticket (gültig für 5 Personen) gelöst und mit 6 Personen genutzt

${ }^{2}$ Rechtlicher Anspruch eines Tagessatzes
} 
In der Regel wird allerdings versucht, den ÖPNV zu meiden. Häufig ist das Fahren ohne (gültigen) Fahrschein, zum Beispiel aufgrund von Schamgefühlen und sozialer Stigmatisierung, ein Geheimnis. 


\section{Zusammenfassung}

Die Motive des Fahrens ohne (gültigen) Fahrschein wurden mit Methoden der qualitativen Sozialforschung untersucht. Der Stand der Forschung zeigt, dass bezüglich der Motive eine Forschungslücke vorhanden ist. Das Ziel des Arbeitspapieres ist es, diese Lücke zu füllen und einen Einblick in ein weitestgehend unerforschtes Feld zu liefern. Ein Verständnis der Beweggründe, die der Fahrt ohne (gültigen) Fahrschein zugrunde liegen, könnte die Perspektive erweitern und Impulse für eine differenzierte Auseinandersetzung mit dem Thema geben.

Zur Untersuchung der Motive wurden insgesamt 31 qualitative Interviews mit Personen durchgeführt, die während einer Fahrkartenkontrolle kein (gültiges) Ticket vorweisen konnten. Die Interviews wurden elektronisch aufgezeichnet, transkribiert und mithilfe der dokumentarischen Methode ausgewertet. Die Auswertung hat eine Motivtypologie ergeben, welche sechs Untertypen in zwei Kategorien enthält: intentionales und nicht-intentionales Fahren ohne (gültigen) Fahrschein.

In der Kategorie intentionales Fahren ohne (gültigen) Fahrschein sind drei Motivtypen enthalten (rationale und opportunistisch, Boykott und Unzufriedenheit und Mobilitätsarmut und soziale Exklusion), bei denen eine bewusste Entscheidung zum Fahren ohne (gültigen) Fahrschein erkennbar wird. Die Kategorie nicht-intentionales Fahren ohne (gültigen) Fahrschein enthält drei Motivtypen, bei denen hingegen keine Absicht erkennbar ist. Es handelt sich um die Typen Personen ohne Tickets, Personen, die ihre Zeitkarten vergessen haben und Personen mit falschen Tickets. Die wichtigsten Merkmale der unterschiedlichen Typen sind in Tabelle 3 zusammengefasst.

Die Untersuchung der Motive des Fahrens ohne (gültigen) Fahrschein ermöglicht einen Einblick in einen noch weitgehend unbekannten lebensweltlichen Bereich. Zudem eignet sich die Motivtypologie zur Erarbeitung motivspezifischer Handlungsoptionen. 
Tabelle 3: Typologie des Fahrens ohne (gültigen) Fahrschein (eigene Darstellung)

\begin{tabular}{|c|c|c|c|c|c|c|c|}
\hline & Typ & Motiv & Personengruppe & ÖPNV-Nutzung & Auslöser & $\begin{array}{c}\text { Einstellung zum } \\
\text { Fahren ohne } \\
\text { (gültigen) } \\
\text { Fahrschein }\end{array}$ & $\begin{array}{l}\text { Strafe und } \\
\text { Straftat- } \\
\text { bestand }\end{array}$ \\
\hline \multirow{3}{*}{$\begin{array}{l}\text { Nicht- } \\
\text { intentional }\end{array}$} & $\begin{array}{l}\text { Personen ohne } \\
\text { Tickets }\end{array}$ & $\begin{array}{l}\text { zweckrational, keine } \\
\text { Intention }\end{array}$ & $\begin{array}{l}\text { zahlende KundInnen } \\
\text { (Kurzstrecke bis } \\
\text { Wochenkarte) }\end{array}$ & $\begin{array}{l}\text { selten oder } \\
\text { unregelmäßig bis } \\
\text { täglich }\end{array}$ & $\begin{array}{l}\text { Monetär und/oder } \\
\text { zeitlich, Wahrnehmung } \\
\text { einer niedrigen } \\
\text { Prüfquote }\end{array}$ & $\begin{array}{l}\text { moralische } \\
\text { Ablehnung }\end{array}$ & $\begin{array}{l}\text { Verständnis } \\
\text { und } \\
\text { Akzeptanz }\end{array}$ \\
\hline & $\begin{array}{l}\text { Personen, die ihre } \\
\text { Tickets vergessen } \\
\text { haben }\end{array}$ & $\begin{array}{l}\text { kein Motiv, keine } \\
\text { Intention }\end{array}$ & $\begin{array}{l}\text { zahlende KundInnen } \\
\text { (diverse Zeitkarten) }\end{array}$ & regelmäßig & Fahrkarte vergessen & $\begin{array}{l}\text { moralische } \\
\text { Ablehnung }\end{array}$ & $\begin{array}{c}\text { Verständnis } \\
\text { und } \\
\text { Akzeptanz }\end{array}$ \\
\hline & 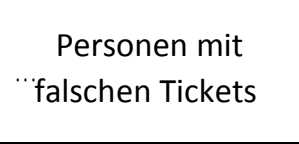 & $\begin{array}{l}\text { kein Motiv, keine } \\
\text { Intention }\end{array}$ & zahlende KundInnen & $\begin{array}{l}\text { selten oder } \\
\text { unregelmäßig bis } \\
\text { täglich }\end{array}$ & Informationsdefizite & $\begin{array}{l}\text { moralische } \\
\text { Ablehnung }\end{array}$ & $\begin{array}{l}\text { Verständnis } \\
\text { und } \\
\text { Akzeptanz }\end{array}$ \\
\hline \multirow{3}{*}{ Intentional } & $\begin{array}{c}\text { rational und } \\
\text { opportunistisch } \\
\text { Handelnde }\end{array}$ & finanzielle Ersparnis & $\begin{array}{c}\text { Personen in finanziell } \\
\text { prekären Situationen und } \\
\text { zweckrational Handelnde }\end{array}$ & $\begin{array}{l}\text { unregelmäßig bis } \\
\text { täglich }\end{array}$ & $\begin{array}{l}\text { Wahrnehmung einer } \\
\text { niedrigen Prüfquote }\end{array}$ & $\begin{array}{l}\text { gerechtfertigt, } \\
\text { logische } \\
\text { Konsequenz }\end{array}$ & $\begin{array}{c}\text { (moralische) } \\
\text { Ablehnung }\end{array}$ \\
\hline & $\begin{array}{l}\text { Boykottierende und } \\
\text { Unzufriedene }\end{array}$ & $\begin{array}{l}\text { Ablehnungsmotive, } \\
\text { politische Motivation }\end{array}$ & alle Personengruppen & $\begin{array}{l}\text { unregelmäßig bis } \\
\text { täglich }\end{array}$ & $\begin{array}{l}\text { Wahrnehmung: hohe } \\
\text { Tarife \& niedrige } \\
\text { Prüfquote }\end{array}$ & Akzeptanz & $\begin{array}{l}\text { (moralische) } \\
\text { Ablehnung }\end{array}$ \\
\hline & $\begin{array}{l}\text { Mobilitätsarme und } \\
\text { sozial Exkludierte }\end{array}$ & Armutsmotive & $\begin{array}{l}\text { Personen in finanziell } \\
\text { prekären oder in } \\
\text { Notsituationen }\end{array}$ & $\begin{array}{l}\text { unregelmäßig bis } \\
\text { täglich }\end{array}$ & $\begin{array}{l}\text { fehlende finanzielle } \\
\text { Ressourcen }\end{array}$ & $\begin{array}{l}\text { moralische } \\
\text { Ablehnung }\end{array}$ & $\begin{array}{c}\text { Verständnis \& } \\
\text { Akzeptanz }\end{array}$ \\
\hline
\end{tabular}




\section{Literaturverzeichnis}

Barabino, Benedetto; Salis, Sara; Useli, Bruno (2013): A modified model to curb fare evasion and enforce compliance: Empirical evidence and implications. In: Transportation Research Part A: Policy and Practice 58, S. 29-39.

Barabino, Benedetto; Salis, Sara; Useli, Bruno (2014): Fare evasion in proof-of-payment transit systems: Deriving the optimum inspection level. In: Transportation Research Part B: Methodological 70, S. 1-17.

Bijleveld, Catrien (2007): Fare dodging and the strong arm of the law. In: J Exp Criminol 3 (2), S. 183-199.

Bohnsack, Ralf (2003): Rekonstruktive Sozialforschung. Einführung in qualitative Methoden. 5. Aufl. Opladen: Leske + Budrich (UTB, 8242: Erziehungswissenschaft, Sozialwissenschaften).

Bonfanti, Gabriele; Wagenknecht, Thierry (2010): Human factors reduce aggression and fare evasion. In: Public Transport International 59, S. 28-32.

Boyd, Kevin; Martini, Christine; Rickard, John; Russell, Allen (1989): Fare Evasion and NonCompliance. In: Journal of Transport Economics and Policy 23 (2), S. 189-197.

Clarke, Ronald V.; Contre, Stephane; Petrossian, Gohar (2009): Deterrence and fare evasion. Results of a natural experiment. In: Security Journal 23 (1), S. 5-17.

Deibel, Lawrence E. (1981): The introduction of European fare collection techniques in the United States. In: Journal of Advanced Transportation 15, S. 55-60.

Delbosc, Alexa; Currie, Graham (2016): Four types of fare evasion. A qualitative study from Melbourne, Australia. In: Transportation Research Part F: Traffic Psychology and Behaviour 43, S. 254-264.

Guarda, Pablo; Galilea, Patricia; Paget-Seekins, Laurel; Ortúzar, Juan de Dios (2016): What is behind fare evasion in urban bus systems? An econometric approach. In: Transportation Research Part A: Policy and Practice 84, S. 55-71.

Killias, Martin; Scheidegger, David; Nordenson, Peter (2009): The Effects of Increasing the Certainty of Punishment: A Field Experiment on Public Transportation. In: European Journal of Criminology 6 (5), S. 387-400.

Lamnek, Siegfried (2010): Qualitative Sozialforschung. Lehrbuch. 5. überarbeitete Auflage. Weinheim, Basel: Beltz.

Misoch, Sabina (2015): Qualitative Interviews. Berlin: De Gruyter Oldenbourg.

Przyborski, Aglaja (2004): Gesprächsanalyse und dokumentarische Methode. Qualitative Auswertung von Gesprächen, Gruppendiskussionen und anderen Diskursen. Wiesbaden: VS Verlag für Sozialwissenschaften (Lehrbuch).

Przyborski, Aglaja; Wohlrab-Sahr, Monika (2008): Qualitative Sozialforschung. Ein Arbeitsbuch. München: Oldenbourg (Lehr- und Handbücher der Soziologie).

Schwerdtfeger, Stefanie; Mehler, Frederik; Wilde, Mathias; Lanzendorf, Martin (2016): Fahren ohne (gültigen) Fahrschein. Stand der Forschung und medialer Diskurs. Hg. V. AG Mobilitätsforschung. Goethe-Universität Frankfurt am Main, Institut für Humangeographie. Frankfurt am Main (Arbeitspapiere zur Mobilitätsforschung, 12). 
Schwerdtfeger, Stefanie; Wilde, Mathias; Lanzendorf, Martin (2017): Dokumentation von BestPractice-Beispielen zum Umgang mit dem Fahren ohne (gültigen) Fahrschein. Hg. v. Arbeitsgruppe Mobilitätsforschung, Institut für Humangeographie, Goethe Universität Frankfurt am Main. Frankfurt am Main (Arbeitspapiere zur Mobilitätsforschung, 14).

Smith, Matha J.; Clarke, Ronald V. (2000): Crime and Public Transport. In: Crime and Justice 27, S. 169-223.

van Andel, Henk (1989): Crime prevention that works: The care of public transport in the Netherlands. In: British Journal of Criminology 29 (1), S. 47-56.

Witzel, Andreas (1982): Verfahren der qualitativen Sozialforschung. Überblick und Alternativen. Frankfurt am Main, New York: Campus Verlag (Campus Forschung, Bd. 322).

Yin, Zhengyu; Xin Jiang, Albert; Tambe, Milind; Kiekintveld, Christopher; Leyton-Brown, Kevin; Sandholm, Tuomas; Sullivan, John P. (2012): TRUSTS: Scheduling Randomized Patrols for Fare Inspection in Transit Systems Using Game Theory. In: Al Magazine 33 (4), S. 59-72. 


\section{Anhang}

\section{Anhang 1: Gesprächleitfaden}

\section{Kontaktaufnahme}

Hallo,

mein Name ist ... und ich möchte Sie im Rahmen meines Dissertationsprojektes gerne zum Thema Fahren ohne (gültigen) Fahrschein interviewen. Die Befragung wird ca. eine halbe Stunde in Anspruch nehmen. Wenn Sie Interesse an einer Befragung haben und diese sofort mit mir durchführen, bekommen Sie als Dankeschön von mir $60 €$ in bar ausgehändigt. Haben Sie Interesse, an der Befragung teilzunehmen?

$\rightarrow$ Bei „,nein" abbrechen und bedanken

$\rightarrow$ Bei ,ja" weiter

Vielen Dank, dass Sie sich bereit erklärt haben an der Befragung teilzunehmen. Das Thema der Befragung ist die Benutzung des ÖPNV ohne gültigen Fahrschein. Ich bin Promotionsstudentin an der Goethe-Universität in Frankfurt und forsche zum Thema Fahren ohne (gültigen) Fahrschein. Innerhalb der Befragung möchte ich herausfinden, warum Sie den ÖPNV ohne (gültigen) Fahrschein benutzt haben. Dabei ist es sehr wichtig, dass Sie mir gegenüber ehrlich sind. Ihre Angaben und Daten werden absolut vertraulich behandelt. Alles was Sie sagen wird ausschließlich anonymisiert verwendet und hat keinerlei rechtli-che oder sonstige Folgen für Sie. Unser Gespräch würde ich gerne aufzeichnen, damit ich es im An-schluss auswerten kann. Wenn Sie bereit sind an der Befragung teilzunehmen und der Tonaufnahme zustimmen, werde ich Ihnen nach erfolgreichem Abschluss des Gespräches $60 €$ in bar auszahlen. Haben Sie Interesse an einer Teilnahme und stimmen Sie der Tonbandaufnahme zu?

$\rightarrow$ Bei „nein" abbrechen und bedanken

$\rightarrow$ Bei ,ja" weiter

Vielen Dank, dass Sie an der Befragung teilnehmen. Ich werde die Tonaufnahme jetzt starten...

Haben Sie noch weitere Fragen?

$\rightarrow$ Bei "ja" auf Fragen eingehen

$\rightarrow$ Bei „nein“" weiter

Wir beginnen jetzt mit dem Interview. 


\section{Einstieg in das Gespräch:}

\section{Fragekategorie: Gründe des Fahrens ohne gültigen Fahrschein}

Situation des Fahrens ohne gültigen Fahrschein schildern lassen

Woher kam die Person?

$>$ Welches Fahrtziel hatte die Person?

$>$ Was war der Wegezweck?

Warum hatte die Person keinen gültigen Fahrschein?

Situationsbedingte Nachfragen

Welche Informationen hatte die Person?

Information: ÖPNV-Benutzung nur mit gültigem Ticket

Information: Ausstellung eines EBE

$>$ Information: Höhe des EBE

$>$ Information: rechtliche Folgen

Fährt die Person häufig/regelmäßig ohne gültigen Fahrschein?

Muster/Strategie?

Bestimmte Strecken?

$>$ Erläuterung 
Fragekategorie: Individuelle Merkmale der eigenen Lebenswirklichkeit

Wie sieht die finanzielle Situation der Person aus?

$>$ Gibt es ein regelmäßiges Einkommen?

$>$ Geht die Person einer Beschäftigung nach?

> Kann die Person gut für sich und die eigenen Familie sorgen?

Wie lebt die Person?

$>$ Mieter/in? Hauseigentümer/in?

Wohnort?

$>$ Arbeitsort?

$>$ Familienstand?

$>$ Kinder?

\section{Fragekategorie: Mobilität und Erreichbarkeiten}

Welche Verkehrsmittel stehen der Person zur Verfügung?

Wie sind die Ziele der Person mit den unterschiedlichen Verkehrsmitteln zu erreichen?

$>$ Arbeitsweg

$>$ Versorgung

$>$ Freizeitwege

\section{Fragekategorie: Einstellung gegenüber dem ÖV/RMV}

Ist die Person auf den ÖPNV angewiesen?

Wie empfindet die Person die Fahrpreisgestaltung?

Wie nimmt die Person Fahrkartenkontrollen wahr?

Häufigkeiten

Akzeptanz? 
Fragekategorie: Einstellung gegenüber Richtig und Falsch

Ist Fahren ohne gültigen Fahrschein in den Augen der Person eine Straftat?

$>$ Ganz wichtig: Begründung?

Findet die Person es okay, dass sie den ÖPNV nutzt und nicht dafür bezahlt?

Was hält das soziale Umfeld vom Fahren ohne gültigen Fahrschein?

$>$ Welchen Einfluss hat das soziale Umfeld auf die Person?

\section{Im Anschluss:}

Für das Gespräch bedanken und weitere Fragen beantworten 
Anhang 2: Fragebogen zur Erhebung soziodemographischer Charakteristika

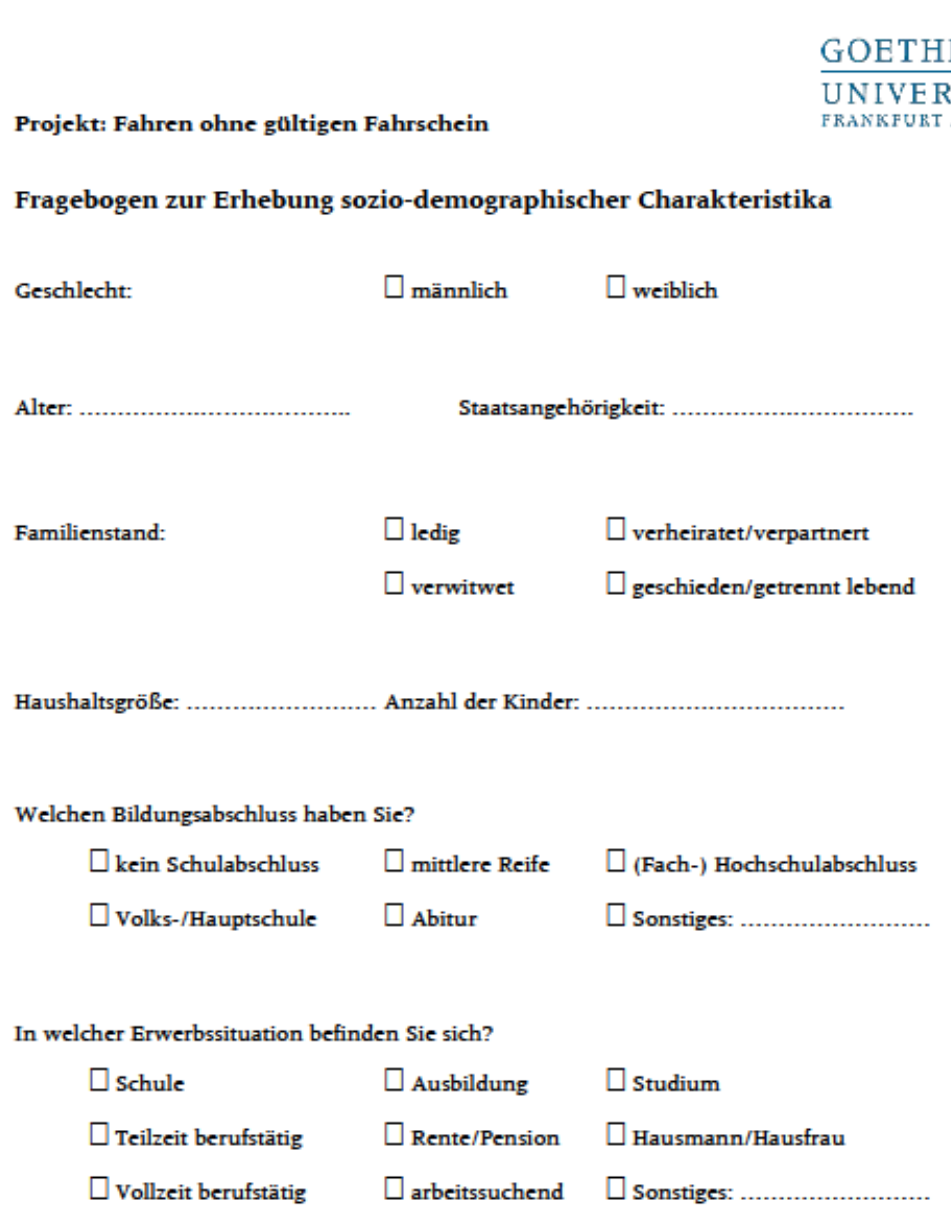

Berufsbezeichnung:

Aktuelles monatliches Nettoeinkommen (Einkünfte nach Abzug von Steuern und Sozialabgaben):
$\square$ unter $500 €$
$\square 2.000$ bis unter $3.000 €$
$\square 500$ bis unter $1.000 €$
$\square 3.000$ bis unter $4.000 €$
$\square 1.000$ bis unter $2.000 €$
$\square 4.000 €$ und mehr
$\square$ Keine Angabe 



\section{Arbeitspapiere zur Mobilitätsforschung}

In den Arbeitspapieren zur Mobilitätsforschung veröffentlichen wir Ergebnisse aus Forschung und Lehre der Goethe-Universität. Online erhältlich unter: http://tinygu.de/Mobilitaet

\section{In dieser Reihe sind folgende Arbeitspapiere erschienen:}

16. Schwerdtfeger, S; Wilde, Mathias; Lanzendorf, Martin (2018): Motive des Fahrens ohne (gültigen) Fahrschein. Arbeitspapiere zur Mobilitätsforschung Nr. 16. Frankfurt a.M.

15. Czowalla, L.; Lanzendorf, M.; Wilde, M.; Fromberg, A.; Gwiasda, P.; Busch, D. (2017): Neuere Entwicklungen zur Integration von Fahrrad und Öffentlichem Verkehr in Deutschland: Überblick zum Stand des Wissens und der Praxis. Arbeitspapiere zur Mobilitätsforschung Nr. 15. Frankfurt a.M.

14. Schwerdtfeger, S.; Wilde, M.; Lanzendorf, M. (2017): Dokumentation von Best-Practice-Beispielen zum Umgang mit dem Fahren ohne (gültigen) Fahrschein. Arbeitspapiere zur Mobilitätsforschung Nr. 14. Frankfurt a.M.

13. Selzer, S.; Kruse, C.; Wilde, M.; Lanzendorf, M. (2016): Integration von Fernbuslinienangeboten. Anforderungen an und Handlungsoptionen für städtebauliche und verkehrliche Integration der Fernbusse in lokale Verkehrssysteme. Ergebnisse einer Fahrgastbefragung in Frankfurt am Main. Arbeitspapiere zur Mobilitätsforschung Nr. 13. Frankfurt a.M.

12. Schwerdtfeger, S.; Wilde, M.; Mehler, F.; Lanzendorf, M. (2016): Fahren ohne gültigen Fahrschein. Stand der Forschung und medialer Diskurs. Arbeitspapiere zur Mobilitätsforschung Nr. 12. Frankfurt a.M.

11. Schubert, S.(2016): Universität in Bewegung. Bestandsanalyse des Verkehrsverhaltens und der Mobilitätseinstellungen von Studierenden und Beschäftigten der Goethe-Universität. Arbeitspapiere zur Mobilitätsforschung Nr. 11. Frankfurt a.M.

10. Blechschmidt, A. (2016): Nischenkonzept oder Zukunftsmodell für nachhaltige Stadtentwicklung? Planungen und Umsetzungen autofreier bzw. autoreduzierter Stadtentwicklungsprojekte im Vergleich. Arbeitspapiere zur Mobilitätsforschung Nr. 10. Frankfurt a.M.

9. Klinger, T.; Deffner, J.; Kemen, J.; Stein, M.; Lanzendorf, M. (2016): Sharing-Konzepte für ein multioptionales Mobilitätssystem in FrankfurtRheinMain. Analyse neuerer Entwicklungen und Ableitung von Handlungsoptionen für kommunale und regionale Akteure. Schlussbericht. Arbeitspapiere zur Mobilitätsforschung Nr. 9. Frankfurt a.M.

8. Schäfer, P. K. et al. (2016): Elektromobilität als Motor für Verhaltensänderung und neue Mobilität. Abschlussbericht des Gesamtvorhabens „Sozialwissenschaftliche und ökologische Begleitforschung in der Modellregion Elektromobilität Rhein-Main“. Arbeitspapiere zur Mobilitätsforschung Nr. 8. Frankfurt. a.M.

7. Prill, T. (2015). Pedelecs als Beitrag für ein nachhaltiges Mobilitätssystem? Eine Analyse zur Akzeptanz, Nutzung und Wirkung einer technologischen Innovation. Arbeitspapiere zur Mobilitätsforschung Nr. 7. Frankfurt a.M.

6. Rolfsmeier, S. (2015): Wohnumzüge und Mobilitätsverhalten. Die Bedeutung von Raumstrukturen und Präferenzen für die Verkehrsmittelnutzung auf Arbeitswegen. Arbeitspapiere zur Mobilitätsforschung Nr. 6. Frankfurt a.M.

5. Belz, M. (2015): Der Wettbewerb „Südtirol radelt“ als erfolgreiche Maßnahme der Radverkehrsförderung?! Eine Evaluation. Arbeitspapiere zur Mobilitätsforschung Nr. 5. Frankfurt a.M.

4. Blechschmidt, A.; Schönduwe, R.; Lanzendorf, M. (2015): Nutzungsmöglichkeiten von regionalen Mobilitätsdaten in der Region Frankfurt Rhein-Main. Regionale Mobilitätserhebungen und Mobilitätskennziffern im Vergleich - Eine Handreichung für die Praxis. Arbeitspapiere zur Mobilitätsforschung Nr. 4. Frankfurt a.M.

3. Schönduwe, R.; Lanzendorf, M. (2015): Nutzung regionaler Mobilitätsdaten -Möglichkeiten zur Kombination und Harmonisierung der regionalen Mobilitätsdaten des Rhein-Main-Panels mit anderen Mobilitäts- und Strukturdaten. Arbeitspapiere zur Mobilitätsforschung Nr. 3. Frankfurt a.M.

2. Belz, M.; Höner, S.; Kruse, C.; Rolfsmeier, S.; Schroer, M. (2014): Mobilitätsmanagement an der Goethe-Universität Frankfurt am Main, Campus Westend. Arbeitspapiere zur Mobilitätsforschung Nr. 2. Frankfurt a.M.

1. Lanzendorf, M.; Schönduwe, R. (2014): Mobilitätsverhalten von Heranwachsenden und Möglichkeiten zur Bindung an den ÖPNV. Arbeitspapiere zur Mobilitätsforschung Nr. 1. Frankfurt a.M.

ISSN: 2363-8133

Arbeitspapiere zur Mobilitätsforschung

Frankfurt a.M. 
ISSN: 2363-8133

Arbeitspapiere zur Mobilitätsforschung Nr. 16 (2018)

Frankfurt a.M. 